\title{
Application of AI and IoT in Clinical Medicine: Summary and Challenges
}

\author{
Zhao-xia LU ${ }^{1}$, Peng QIAN ${ }^{1}$, Dan BI ${ }^{1}$, Zhe-wei YE², Xuan $\mathrm{HE}^{3}$, Yu-hong ZHAO ${ }^{4 *}$, Lei SU ${ }^{1}$, Si-liang $\mathrm{LI}^{1}$, Zheng-long ZHU ${ }^{1}$ \\ ${ }^{1}$ Neusoft Hifly Medical Technology Co., Ltd, Shenyang 110179, China \\ ${ }^{2}$ Department of Orthopedics, Union Hospital, Tongji Medical College, Huazhong University of Science and Technology, Wuhan \\ 430022, China \\ ${ }^{3}$ College of Medicine \& Biological Information Engineering, Northeastern University, Shenyang 110169, China \\ ${ }^{4}$ Department of Clinical Epidemiology, Clinical Research Center, Shengjing Hospital, China Medical University, Shenyang \\ 110004, China
}

(C) Huazhong University of Science and Technology 2021

\begin{abstract}
[Abstract] The application of artificial intelligence (AI) technology in the medical field has experienced a long history of development. In turn, some long-standing points and challenges in the medical field have also prompted diverse research teams to continue to explore AI in depth. With the development of advanced technologies such as the Internet of Things (IoT), cloud computing, big data, and $5 \mathrm{G}$ mobile networks, AI technology has been more widely adopted in the medical field. In addition, the in-depth integration of AI and IoT technology enables the gradual improvement of medical diagnosis and treatment capabilities so as to provide services to the public in a more effective way. In this work, we examine the technical basis of IoT, cloud computing, big data analysis and machine learning involved in clinical medicine, combined with concepts of specific algorithms such as activity recognition, behavior recognition, anomaly detection, assistant decision-making system, to describe the scenario-based applications of remote diagnosis and treatment collaboration, neonatal intensive care unit, cardiology intensive care unit, emergency first aid, venous thromboembolism, monitoring nursing, image-assisted diagnosis, etc. We also systematically summarize the application of AI and IoT in clinical medicine, analyze the main challenges thereof, and comment on the trends and future developments in this field.
\end{abstract}

Key words: artificial intelligence; Internet of Things; big data; cloud computing; clinical medicine

The World Health Organization points out that the global health manpower shortage is still severe and that the health of the global population will be seriously affected if the problem is not addressed in a timely manner ${ }^{[1]}$. At present, the total number of nurses in the world is 27.9 million and the employment gap is as high as 5.9 million, which far from meets the global demand for medical services ${ }^{[2]}$. According to a study published in the Lancet, more than 3.6 million people die each year from lack of medical resources in lowand middle-income countries ${ }^{[3]}$.

\section{RESEARCH BACKGROUND AND SIGNIFI- CANCE}

In 2019, the global population aged 65 and over amounted to 703 million, and the number of elderly people is expected to double to 1.5 billion by $2050^{[4]}$. The elderly are a population with a high incidence of disease, usually coexisting diseases, and their demand for medical services is much higher than that of the general population. Some survey data suggest that $80 \%$

Zhao-xia LU, E-mail: luzx@neusoft.com

\#Corresponding author, E-mail: zhaoyuhong@sj-hospital.org of adults aged 65 and older have at least one chronic disease and that $68 \%$ have two or more ${ }^{[5]}$. For example, nearly two-thirds of the elderly in the United States and Germany have comorbidities ${ }^{[6,7]}$, about threequarters of those over the age of 75 in Australia have comorbidities $^{[8]}$, and nearly $1 / 5$ of the elderly in China suffer from at least two chronic diseases ${ }^{[9]}$. All of these scenarios negatively affect the health system around the world.

In 2020, a sudden COVID-19 pandemic swept across more than 200 countries around the world. The surge of critically ill patients in a short period of time forced many countries' medical resources to be stretched and overwhelmed, which posed a tremendous impact on the global health system. In the post-epidemic era, we are still faced with multiple unavoidable challenges, such as an insufficient supply of medical resources, an uneven distribution of high-quality medical resources, an imperfect medical service system, and insufficient medical and health service quality.

The breakthrough and development of newgeneration information technologies such as artificial intelligence (AI), Internet of Things (IoT), and big data provide the best option to solve the above-mentioned problems. The biggest advantage of AI in the medical 
field is its accuracy and efficiency, which helps doctors make more suitable decisions and thereby greatly improves the standard of diagnosis and treatment and improves the efficiency and quality of medical services. With the advantages of comprehensive perception, reliable transmission and intelligent processing, IoT can gather, integrate, classify and exchange realtime data on a large scale around the clock, and realize dynamic perception, comprehensive coverage, ubiquitous connection and the mobile interconnection of the health care system. Big data analysis technology provides high-quality data sets for AI's continuous training and optimization of algorithm models, which enable clinical decision support systems to be more intelligent.

In the past few decades, countries have actively explored the application of AI, IoT and other new technologies in the medical field, and have constantly driven the innovation and development of the medical industry. In terms of medical collaboration, AI, IoT and high-definition video technology are used to realize bedside vital sign monitoring, remote ward rounds, etc. For example, in the COVID-19 pandemic, the combination of 5 th Generation Mobile Communication Technology (5G), IoT and audio and video technology has enabled critical medical experts in different locations to communicate with bedside medical staff in two-way audio and video in real time, view patients' vital signs and patients' electronic medical records and other clinical data in real-time, guide medical staff to make clinical decisions quickly and accurately, and demonstrate its significant role in scientific and technological anti-pandemic effects ${ }^{[10-12]}$. In the aspect of critical treatment, IoT is used to collect and display the vital sign data of various types of medical equipment connected by critically ill patients, and to configure collection items and early warning items individually, so as to facilitate doctors and nurses to monitor patients' vital signs in real-time, realize intelligent alarm after exceeding the critical value of patients' signs, predict patients' life-threatening risks, accurately analyze the best treatment time, and adjust the treatment plan in time ${ }^{[13]}$. In terms of emergency services, $5 \mathrm{G}$ and IoT technology is used to realize unified management of patients' vital sign information, audio and video information and pre-hospital first aid electronic medical record information in the transfer stage of pre-hospital first aid. These techniques help realize the concept of admission "as soon as you get on the bus". At the same time, AI technology helps achieve intelligent scheduling, intelligent triage and accurate auxiliary diagnosis to seize prime opportunities to improve the survival rate of critically ill patients ${ }^{[14]}$. In the field of medical imaging, deep learning algorithms have been widely used in different modalities, such as CT, MRI, PET, and ultrasound, as well as the application of tumor detection, segmentation, disease prediction, etc ${ }^{[15]}$. As a result, excellent results have been achieved in the fields of lung nodule detection ${ }^{[16]}$, diabetic retinopathy (DR) screening ${ }^{[17]}$, and the accurate diagnosis and treatment of cardiovascular diseases ${ }^{[18]}$.

This paper summarizes the relevant technical basis of AI and IoT in terms of their applications in clinical medicine, analyzes the main challenges thereof, and discussed various ideas and opinions for future research.

\section{TECHNICAL BASIS}

The application of AI in clinical medicine mainly involves IoT, cloud computing, big data analysis, machine learning, etc. The following sections briefly describe the definition of each technology and the related core content.

\subsection{Technical Framework}

The technical framework involved in the application of AI in clinical medicine is shown in fig. 1 . According to this figure, $\mathrm{AI}$ applications can be divided into three levels from bottom to top, as follows: (1) the basic layer, which mainly includes IoT and cloud computing; (2) the data layer, which mainly includes basic data, business data, subject data, etc.; and (3) the data analysis layer, which mainly includes big data analysis and machine learning.

\subsection{Internet of Things}

Io $\mathrm{T}$ is an important component of the new generation of information technology. IoT applies a variety of information sensing devices to detect in real-time any object or process that needs to be monitored and combines them across the internet to form a large integrated network. Its purpose is to achieve a connection between a variety of objects and people to facilitate their identification, management and control. The core and foundation of IoT is still the internet, which extends and expands the client and his/ her associated network to many goods and objects and exchanges and communicates information ${ }^{[19-21]}$.

IoT technology is integrated by a variety of technologies, among which sensor networks, radio frequency identification (RFID) and quick response (QR) codes allow devices to obtain data from the physical world. Solutions such as Bluetooth, wireless local area network (WLAN), and the internet provide channels for data transmission. The key technologies involved in the implementation, application and research of IoT mainly involve a perception layer, network layer and application layer. The application layer is the final component that uses IoT to obtain data. The technologies required for IoT focus on the perception layer and the network layer. In this regard, the sensing layer involves QR code technology, RFID, sensor technology, etc. By contrast, the general 


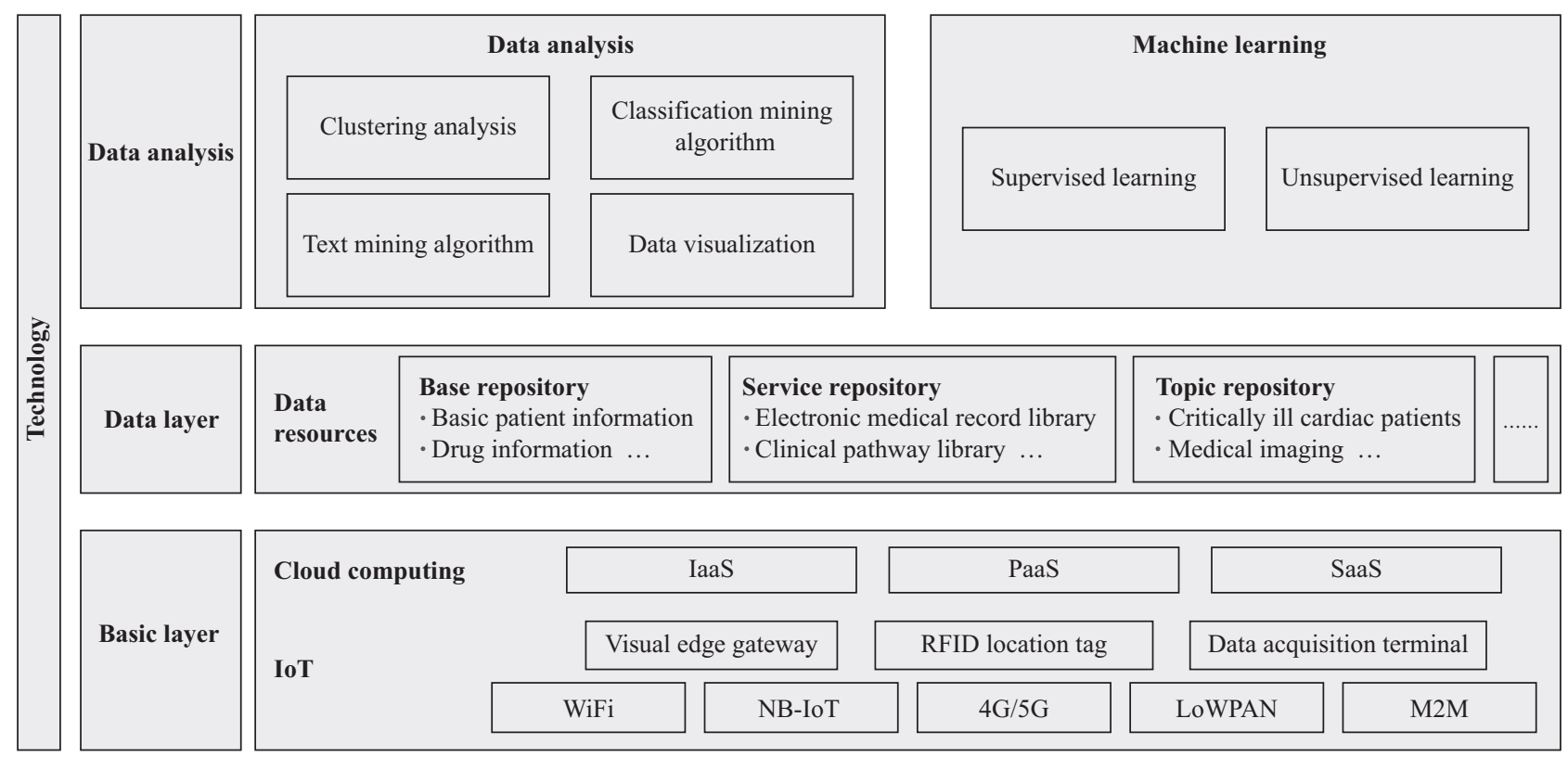

Fig. 1 Technical framework of the application of AI in clinical medicine

AI: artificial intelligence; IaaS: Infrastructure as a Service; IoT: internet of things; LoWPAN: low power wireless personal area network; M2M: machine to machine; NB-IoT: narrow band IoT; PaaS: Platform as a Service; RFID: radio frequency identification; SaaS Software as a Service

technologies of the network layer include WiFi, narrow band IoT (NB-IoT), Zigbee, mobile communication technology $(4 \mathrm{G} / 5 \mathrm{G}$, etc.), low power wireless personal area network (LoWPAN) and machine to machine (M2M) technology, etc ${ }^{[22]}$. Among these, $5 \mathrm{G}$ is a new generation mobile communication network that involves a high speed and low delay Dalian connection. $5 \mathrm{G}$ therefore greatly promotes the development of IoT and better meets the needs of IoT applications, including mobile medical and emergency needs ${ }^{[23]}$.

IoT serves a vital role in the medical industry, especially as $5 \mathrm{G}$ empowers the existing medical service system, and enables medical staff, patients, and hospital managers to work smarter, more accurately, and more actively. This allows clinical care to develop rapidly toward mobile, intelligent, and visualized modes, while also improving service capabilities and management efficiency with telemedicine, emergency and first aid. IoT also facilitated new application scenarios such as diagnosis and treatment collaboration and intensive care based on $5 \mathrm{G}$ converged networks ${ }^{[24]}$.

\subsection{Cloud Computing}

Cloud computing is a type of distributed computing that decomposes the huge data computing processing programs into countless mini programs through the "cloud" network. The cloud then processes and analyzes these mini programs through a system composed of multiple servers to obtain the results and return them to users. Cloud computing is a new computing paradigm that aims to provide end users with a reliable, customized and dynamic computing environment with quality of service assurance. In general, cloud computing service types are divided into three categories, namely Infrastructure as a Service (IaaS), Platform as a Service (PaaS) and Software as a Service $(\text { SaaS })^{[25,26]}$.

The implementation of cloud computing requires a variety of technologies ${ }^{[27]}$. Virtualization technology provides a virtualized information technology (IT) infrastructure on demand and allows users to access cloud resources through a customized network environment. This is the foundation of cloud computing since it provides flexible and scalable hardware services. Service process and workflow technology automatically arrange services from different sources and types by providing a complete set of service templates to form a transparent and dynamic service flow or workflow transparently for users. Service-oriented architecture (SOA) technology and cloud computing services are usually exposed as web services, which need to follow industry standards such as web service description language (WSDL) and universal description discovery integration (UDDI). Network storage technology and programming models use network storage technology to provide storage capacity for users to rent, and provide users with simple cloud programming models to adapt to the cloud infrastructure.

Cloud computing makes it possible to provide medical services with high efficiency and low cost, and helps to improve the quality of patient services. For example, the use of cloud computing can achieve data exchange and sharing between different systems, achieve mutual recognition of test results, provide strong support for remote consultation, enlarge high- 
quality medical resources, and alleviate the difficulty of seeing a doctor ${ }^{[28]}$.

\subsection{Big Data Analysis}

The purpose of big data analysis is to extract knowledge, help make predictions, identify trends, find hidden information, and make decisions ${ }^{[29]}$. Data mining technologies mainly include clustering analysis, classification mining algorithms, text mining algorithms and data visualization techniques.

Cluster analysis divides data into different groups according to some measure of closeness. This is an exploratory analysis that functions by giving a classification rule in advance and can automatically classify sample data. The classification mining algorithm aims to establish a map from the input data to the classification labels. Classification mining algorithms mainly include a decision tree ${ }^{[30]}$, Bayesian inference ${ }^{[31]}$, and artificial neural networks $(\mathrm{ANNs})^{[32]}$. Decision tree learning is a case-based inductive learning algorithm, which focuses on inferring the classification rules expressed in the form of decision tree from a group of disordered and irregular cases. Bayesian inference is a graphical pattern technique used to represent the connection probability between variables, and provides a natural method to represent causal information to discover the potential relationship between data. An ANN is a parallel nonlinear dynamic system model, which is a mathematical model for information processing similar to the structure of synaptic connections in the brain. The text mining algorithm ${ }^{[33]}$ is a process of obtaining users' interested or useful patterns from unstructured text information, and extracting previously unknown, understandable and ultimately available knowledge from a large number of text data. This approach mainly includes text preprocessing, text word segmentation, text mining, semantic analysis, etc. Data visualization technology ${ }^{[34]}$ refers to the theory, methodology and technology of using computer graphics and image processing technology to convert data into graphics or images, and to interactively display and process them.

Big data analysis technology can play an important role in disease prevention, diagnosis and treatment, clinical scientific research, and drug application effect comparison $^{[35,36] \text {. }}$

\subsection{Machine Learning}

Machine learning is a special data-driven analysis method, which can build models automatically in order to find statistical patterns in high-dimensional and multivariate data sets. The theme of machine learning technology is pattern recognition, which provides support for the prediction and decision-making process of diagnosis and treatment planning ${ }^{[37,38]}$.

There are two main types of machine learning. The first method is supervised learning, which trains algorithms using specific examples. The machine receives a certain number of inputs and correct outputs, and learns to identify errors by comparing empirical results with the correct outputs. This way of learning is used when history can be used to predict future events and mainly focuses on classification and regression. Another method is unsupervised learning, where the machine must actively explore the data through a development model to discover hidden patterns in the data without being informed of any correct output. This method is often used to locate and distinguish outliers, and mainly focuses on clustering methods ${ }^{[20]}$.

In the medical field, structured data accounts for about $20 \%$ of the current electronic medical records, and the other $80 \%$ is made up of unstructured data. In addition, the nature of medicine is a narrative, which implies that machine learning technology in the medical field needs to focus more on the relationship between organizing and establishing a large amount of unstructured raw data, and to use and understand such data on a large scale. This will be very beneficial to the medical field and may have a wide range of implications ${ }^{[20]}$.

\section{ALGORITHMS AND METHODS}

The related applications of AI and IoT in clinical medicine are mainly related to activity identification, behavior recognition, anomaly detection, auxiliary decision-making, anonymization, privacy protection and other technical fields. The following is a brief description of the core algorithms and methods involved in various technical fields.

\subsection{Activity Recognition}

In clinical medicine, inertial sensors are mainly used to monitor the activities of patients using activity recognition technology. The main processing steps include preprocessing, segmentation, feature extraction, reduction and classification. Each step involves distinct algorithms and methods to properly execute $^{[39]}$.

3.1.1 Preprocessing The data obtained by a sensor must first be preprocessed to remove high-frequency noise within the data. the methods that can be used include nonlinear, low-pass median, Laplace and Gaussian filters, etc. While useful information is retained, the original data still need to be represented. Common methods to do this include the following: Piecewise Linear Representation (PLR); Fourier Transforms (FTs), which can retain the original information and also reduce the dimension of data; and Wavelet Transforms (WTs), which can better represent functions with discontinuity and spikes. Among these methods, the Discrete-WT (DWT), where the wavelet is discretely sampled, is a powerful method to identify the conversion between gestures and to eliminate the noise in activities such as walking and running. 
3.1.2 Segmentation The difficulty of continuous activity recognition is to extract important and useful information from continuous sensor data streams. Therefore, it is necessary to adopt a segmentation method of time series data, which can be in the form of sliding window, top-down, bottom-up, sliding window and bottom-up. Among these options, the sliding window algorithm is simple, intuitive and online, and is most popular in the medical field. This approach starts with a small time series and adds new data points until the fitting error of the potential segment is greater than a user-defined threshold.

3.1.3 Feature Extraction The main feature types that can be extracted are as follows: time domain features, frequency domain features, time-frequency domain features, heuristic features, domain-specific functions, etc. Time domain features are extracted directly from a data segment, and include basic waveform features and signal statistics. The frequency domain characteristics focus on the periodic structure of the signal, which can be used to capture the periodicity of acceleration data in the frequency domain and can distinguish sedentary activity from strenuous activity. Time-frequency domain characteristics usually use wavelet technology to study the time-frequency characteristics of signals, and are mainly used to detect the transition between different activities. Domain-specific functions require functions tailored to specific applications, such as Time-Domain Gait Detection, which utilizes a timedomain algorithm for gait detection in addition to the Fast Fourier Transform (FFT). As a result, the accuracy of step size detection can reach $95 \%$.

3.1.4 Dimensionality Reduction Dimensionality reduction generally includes feature selection and feature transformation. The purpose of feature selection is to select features with the strongest recognition ability and the greatest contribution to the performance of the classifier. This procedure will ultimately create a subset of the existing features and may be discovered using support vector machine-based feature selection, k-means clustering, Forward-Backward sequential search, etc. Feature transformation technology aims to map high-dimensional feature space to a much lower dimension, resulting in fewer original feature combinations. Effective methods for this include principal component analysis (PCA), independent component analysis (ICA), local discriminant analysis (LDA), etc.

3.1.5 Classification and Recognition Thresholdbased techniques are widely used to distinguish activities of different intensities. By contrast, pattern recognition technologies include decision tables, decision trees, Nearest Neighbor (NN), Naïve Bayes (Naïve Bayes, NB), Support Vector Machines (SVMs), Hidden Markov Models (HMMs), Gaussian Mixture Models (GMMs), etc.

\subsection{Behavior Recognition}

Behavior recognition has always been a hot topic in computer vision research, and is also a key technical element in clinical applications. In intensive care, emergency rescue, rehabilitation and other scenarios, it can monitor the behavior of patients through remote video recording to effectively identify the movements of patients and provide a basis for treatment and evaluation. At present, the main methods of behavior recognition use a convolution neural network $(\mathrm{CNN})$ or recurrent neural networks (RNN). The classic CNNs include AlexNet and VGG16, which can extract different features through various permutations and combinations of convolution, pooled and fully connected layers ${ }^{[0]}$. An RNN takes a piece of sequence data as the input, recurses in the changing direction of the sequence, and circulates the unit according to the chain connection ${ }^{[4]}$. Nowadays, most of the existing behavior recognition algorithms based on deep learning are developed on the basis of a double-stream network, 3D CNN and RNN, especially long short-term memory (LSTM).

For the deep learning algorithm of double-stream network-based behavior recognition, the object of behavior recognition is more video. Compared with a single picture, this algorithm needs to add time series information. Therefore, the double-flow convolution network learns the characteristics of spatial flow and time flow separately, and finally integrates them to obtain the recognition result. This method makes up for the lack of time flow characteristics in traditional methods. At the same time, the double-flow network can further subdivide the time flow into local time flow and global time flow, resulting in a three-flow network that can improve the robustness of spatio-temporal information and the accuracy of recognition results ${ }^{[42,43]}$.

In the 3D convolution-based behavior recognition deep learning algorithm, the 3D convolution results include height, width, and time dimensions. The optimal $3 \mathrm{D}$ convolution kernel size is recognized as $3 \times 3 \times 3$, and the network is designed with 8 convolution layers. 3D convolution includes the time dimension and can handle video data well, but the parameter settings are very complicated. Therefore, another P3D residual network (Pseudo-3D ResNet) that reduces network parameters can be used by employing ResNet's network framework with a $1 \times 3 \times 3$ convolution (to obtain spatial dimensional features) and a $3 \times 1 \times 1$ convolution (acquisition of time dimension features) in a cascade or in parallel instead of the $3 \times 3 \times 3$ original $3 \mathrm{D}$ convolution. At the same time, a 3D/2D hybrid convolution module (MiCT) can also be used to process video data. The MiCT module increases the depth of the 3D CNN, can generate more advanced 3D features, and reduces the complexity of learning $3 \mathrm{D}$ features and spatiotemporal feature fusion ${ }^{[44-46]}$. 
In the LSTM network-based behavior recognition algorithm, the cyclic network module of an RNN can not only learn the current information, but also save the previous time series information. However, compared to the information with longer time series, an RNN is prone to the problem of gradient disappearance. At this time, an LSTM network can be used to solve this problem as it replaces the hidden layer nodes in the original RNN model with a memory unit and utilizes cell states to store historical information. Therefore, three gate structures are designed to update or delete the information in the cell state through a sigmoid function and point-by-point product operation ${ }^{[47,48]}$.

\subsection{Abnormal Detection}

The purpose of abnormal detection is that some data that are inconsistent with the rest of the data or do not follow the expected trends or characteristics ${ }^{[49]}$. Abnormal detection helps to identify abnormal behavior in medical treatment so as to improve the safety thereof. The main techniques of abnormal detection can be divided into supervised learning and unsupervised learning.

Supervised learning needs labeled data to create a training set and then uses a support vector machine or similar classifier to effectively perform supervised learning. However, in practice, the tagged data used for abnormal detection is rarely available and it is difficult to cover all possible exceptions that may occur in a domain. This often results in unsupervised learning being the most widely used for abnormal detection problems. Unsupervised learning is of special importance in unknown disease detection, new drug discovery, fault diagnosis and identification. Abnormal detection algorithms using unsupervised learning can be divided into distribution-based, depth-based, distance-based, clustering-based and density-based algorithms. The relatively simple unsupervised method is to use clustering algorithm to identify objects in small clusters or objects that are not assigned to any clusters. Based on the premise that exceptions occur in sparse neighborhoods, some unsupervised techniques are based on the distance between the instance and the nearest neighbor. The commonly used proximity algorithms are the approximate $\mathrm{K}$-nearest neighbors (AKNN) method, reverse K-nearest neighbor (KNN) and local outlier factor. Other popular methods include statistical and probabilistic analyses. The abnormal detection method includes two steps: outlier analysis, score allocation and score transfer ${ }^{[4,50]}$.

\subsection{Auxiliary Decision-Making}

Auxiliary decision-making is used to improve the speed and accuracy of doctors' diagnoses. It uses data mining technology to establish a classification model based on medical multi-dimensional data, and provides the corresponding diagnosis according to the new patient symptoms ${ }^{[51]}$.
The prediction tasks of auxiliary decisionmaking can be divided into two types: predictive and descriptive. The predictive task is to infer a function from a set of marked training samples by mapping data samples based on both the input and output (supervised learning). For example, a neural network, classification/ regression tree and support vector classifier/regression are supervised learning algorithms. By contrast, descriptive tasks attempt to infer untagged input data, such as clustering and association techniques. They group objects into clusters or find some interesting associations between variables in the database. These techniques include k-means clustering, hierarchical clustering and frequent itemset rule extractor. In these methods, clinical data classification is the key, which needs to be selected from ensemble, decision tree, rulebased, Bayesian and neural network classifiers ${ }^{[52]}$.

The C4.5 decision tree algorithm is a common classification algorithm with a main goal of building a decision tree from examples and their data sets. The algorithm follows the divide-and-conquer model, and each step tries to find the best attribute for segmenting the data set by calculating the entropy and information gain. Currently, a variety of data mining techniques have been used to diagnose a variety of diseases, including heart disease, diabetes, lung cancer, etc. In SVMs, KNNs and neural networks, C4.5 exhibits the highest accuracy. At the same time, compared with Bayesian networks, J48 exhibits a higher performance accuracy. Therefore, the decision tree is the most accurate classification model in medical applications ${ }^{[33]}$.

\subsection{Anonymization and Privacy Protection}

The basic information of patients, test results, diagnostic information and prescription information all belong to the category of private information. This information cannot be identified by other users without authorization, and therefore privacy protection technology needs to be used in data sharing to ensure the security of patient data.

At present, privacy protection technology that is implemented all over the world is mainly the "anonymous" method. K-anonymous can prevent the disclosure of identifying information, but cannot prevent the disclosure of attribute information. L-diversity is a group-based anonymous form that protects privacy in the dataset by reducing the granularity of the data representation. It is an extension of the k-anonymous model, which uses methods such as generalization and suppression to reduce the granularity of the data representation so that any given record is mapped to at least $\mathrm{k}$ different records in the data ${ }^{[53]}$. T-closeness is a further improvement of the anonymization of the L-diversity group and also extends the L-diversity model. This method distinguishes the value of the attribute by considering the distribution of the value of the attribute, and exhibits a primary advantage in that it 
intercepts the exposure of the attribute.

In order to prevent an attack and reveal personally private information from published data, a differential privacy protection method based on fog computing can be adopted. A data publishing algorithm based on this was developed using MaxDiff histogram technology. Differential privacy is realized by adding Laplacian noise to the dataset. Even if the attacker has strong background knowledge, the owner can protect personal privacy from invasion and can thus improve the efficiency of big data for protecting personal privacy ${ }^{[54]}$.

To address the problem of data privacy protection in the process of machine learning and deep learning, differential privacy has improved and involves three privacy protection methods which include anonymizing first, learning first and anonymized learning. In the anonymizing first method, k-anonymous technology, followed by a machine learning algorithm, is used to anonymize the database. The learning first method first learns the data through a machine learning algorithm and then adds differential privacy noise to the results. In the anonymized learning method, each parameter in the machine learning model is anonymous ${ }^{[54]}$.

\section{CLINICAL APPLICATIONS}

The in-depth integration of AI and IoT technologies enables the medical industry to become more intelligent and humane. This section summarizes and explains typical AI and IoT applications in clinical medicine.

\subsection{Remote Diagnosis and Treatment Collaborations}

In recent years, the advantages of remote diagnosis and treatment collaborations in optimizing the allocation of medical resources, reducing medical costs and improving the quality of medical services have become increasingly prominent. This focus has become a popular direction for medical development, and is also one of the important measures used to solve the problem of unequal allocation of medical resources $^{[55]}$.

Remote diagnosis and treatment collaborations, commonly known as remote consultation or remote medical consultation, refer to a kind of medical diagnosis that provides medical information through modern communication tools, computers and network technology to realize cross-regional medical diagnosis. It further formulates and improves the treatment plan, and through the integration of high-quality medical resources, ensures that patients receive an effective and standardized diagnosis and treatment plan ${ }^{[55]}$.

Under the guidance of future trends, the dependence of the medical industry on IoT is converging on remote diagnosis and treatment collaborations. This collaboration can collect, record and analyze new data streams faster and more accurately by integrating resources such as computers, communication networks, medical technologies and devices, and by collecting and sharing information through the cloud ${ }^{[56]}$. Doctors are allowed to log into the telemedicine consultation system to designated beds through the internet, enabling patients to interact with medical experts and grass-roots medical staff in high-definition audio and video in a face-to-face way. This technology also allows doctors to consult patients about their illness and then share data in a secure environment. This solves the problem of uneven distribution of medical resources, breaks through the geographical barriers for patients to receive medical care, reduces the chances of infection and radiation among medical personnel ${ }^{[57]}$, improves the efficiency of ward rounds, realizes the reduction of high-quality medical resources, develops visits to diagnosis, treatment, nursing and other medical services, improves the level of primary health care, and reduces health care costs.

The collaborative model has been applied in many medical fields, such as the medical imaging cloud platform, which can centrally store image data uploaded by hospitals and various cooperative medical institutions to realize the sharing of image data information across these institutions. At the same time, this network builds a remote image consultation platform with the central hospital as the main body, realizes the auxiliary recognition of medical images with the help of AI technology, constructs a super multi-disciplinary joint diagnosis and treatment model, and provides services that include remote consultation, remote guidance and remote training for grass-roots hospitals. This in turn enhances the accuracy of diagnosis and treatment, and improves the level of primary medical care. Fig. 2 is a schematic diagram of the remote image consultation platform with the central hospital as the main body.

With the rise of AI and IoT technology, telemedicine will gradually break through the limitations of current technology, allowing anyone to obtain quality and valuable medical services anywhere in the world. In the future, remote diagnosis and treatment collaboration will be available everywhere.

\subsection{NICU Scenario Application}

In 2019, data released by the United Nations Children's Fund showed that $46 \%$ of deaths among children under the age of five occurred during the neonatal period ${ }^{[58]}$. With the improvement of obstetrics and neonatal intensive care treatment technology, the survival rate of premature infants, especially very low and ultra-low birth weight infants, has increased significantly. As a result, problems such as brain damage, infection, and breathing impairment of premature infants have gained more attention. How to predict early neonatal-related high-risk symptoms has evolved into the center of neonatal intensive care unit (NICU) 


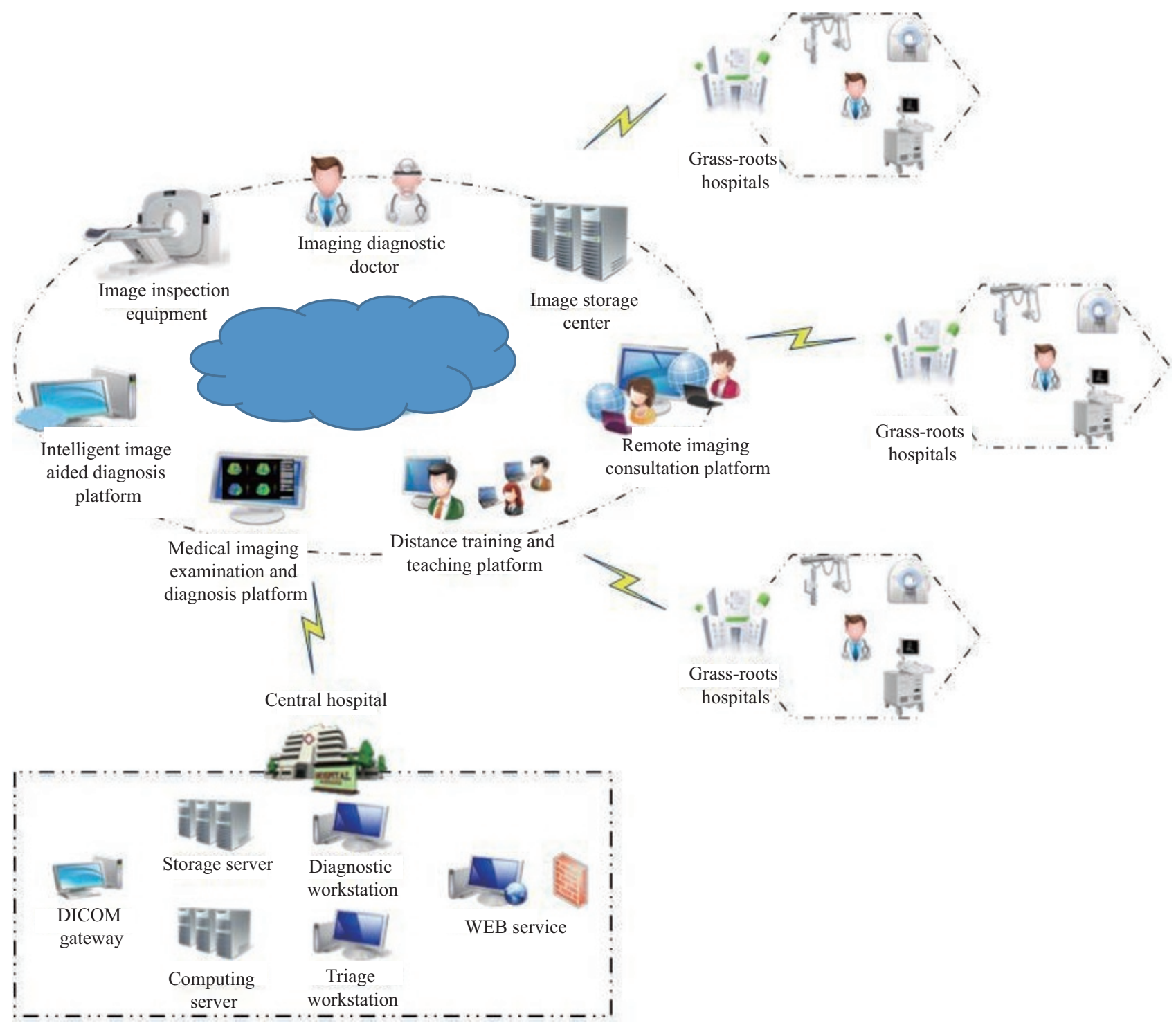

Fig. 2 Schematic diagram of a remote imaging consultation platform with a central hospital as the main body

work. However, how to implement standardized, accurate, and evidence-based management in neonatal intensive care medicine and how to conduct in-depth clinical research are issues that need to be discussed by neonatologists. In recent years, the digital-driven clinical research paradigm has brought new ideas to clinical medicine ${ }^{[59,60]}$.

Based on the new concepts of big data, machine learning and AI technology, the digital-driven clinical research paradigm explores the correlation between data by obtaining the relevant information, algorithms and models of the research as comprehensively as possible. It is mainly reflected in two aspects, namely the in-depth development and utilization of multivariate data and the AI-aided clinical decisionmaking system based on multi-dimensional data. In order to make full use of a large number of real-world patient information, it is necessary to collect, process and apply the data through medical IoT and other information technologies. AI-assisted interpretation of medical images, electroencephalograms (EEG), pathological pictures and fundus images can correctly carry out early warning and diagnosis of relevant diseases. Besides, the diagnosis and treatment decision-making system combined with medical history, pathology, imaging and patient outcomes can recommend individualized treatment plans and predict treatment prognosis for related diseases. Therefore, in neonatal critical medicine, we can learn from the success of the above-mentioned medical field ${ }^{[61,62]}$.

There are massive and multi-dimensional clinical data in the NICU, which suggests that the digitaldriven clinical research paradigm is helpful to realize the rapid and accurate diagnosis and treatment of critical newborns. For critical newborns, in addition to the collection of some routine symptoms and signs, the dynamic monitoring data of the instrument and the parameters of different developmental stages are also very important in clinical decision-making. Such important parameters include real-time vital sign data, 
blood biochemical data, EEG and imaging data. Fig. 3 shows the monitoring of the vital signs of newborns during sleep using physiological radar monitoring systems (PRMS) based on IoT technology ${ }^{[63]}$.

Clinical medicine is entering a digital age. The NICU is an important practical field to realize digital medical treatment. Combined with the real-time vital sign data of patients obtained by medical IoT, the data of electronic medical record systems and all kinds of imaging data can help machine learning and AI methods to actively explore the unknown of neonatal critical medicine ${ }^{[64,65]}$.

\subsection{CCU Scenario Application}

The cardiology intensive care unit (CCU) mainly treats critically ill patients with heart disease. In the

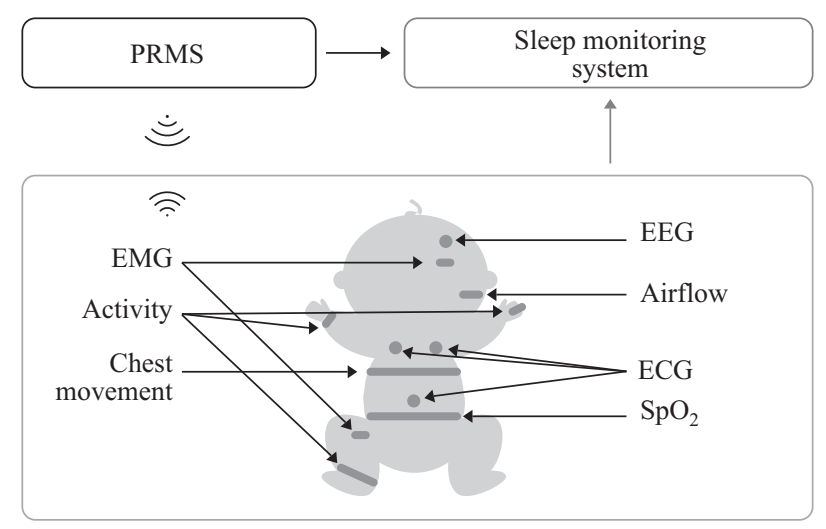

Fig. 3 Monitoring of newborn's vital signs based on IoT technology PRMS: physiological radar monitoring systems; EMG: electromyography; EEG: electroencephalogram; ECG: electrocardiogram treatment process, emerging technologies urgently need to be introduced to quickly and accurately identify the risk factors of critically ill cardiac patients, and then to help doctors make prognostic predictions and medical decisions.

In terms of the combination of $\mathrm{AI}$ and IoT, Kannathal et al ${ }^{[66]}$ adopted neural network algorithm to collect ECG data from ECG machines through IoT for analysis and diagnosis. This work showed that these signals can be divided into normal, abnormal, and critical groups, with an accuracy of $99 \%$, which is sufficient to identify critically ill heart patients. Fig. 4 shows the grading system for patients with heart disease. Sengupata et al ${ }^{[67]}$ applied a cognitive computing method to identify and diagnose constrictive pericarditis and restrictive pericarditis by means of IoT and echocardiography data collected from ultrasound equipment.

The prognosis of critical heart disease is more complicated than that of ordinary heart disease. The use of AI and IoT technology to monitor patients and provide feedback plays an important role in the rehabilitation of patients with critical heart disease. Schoenrath et a ${ }^{[68]}$ introduced an AI-assisted Lokomat system to train patients with "gait therapy" after cardiac surgery. The system can generate an exercise plan according to the condition of patients and feedback from the effect of exercise. At the same time, the program did not increase the risk of knife edge infection, and provided a safer and effective exercise rehabilitation method for patients after cardiac surgery. Ottaviano et al[ ${ }^{[69]}$ developed a cardiac rehabilitation

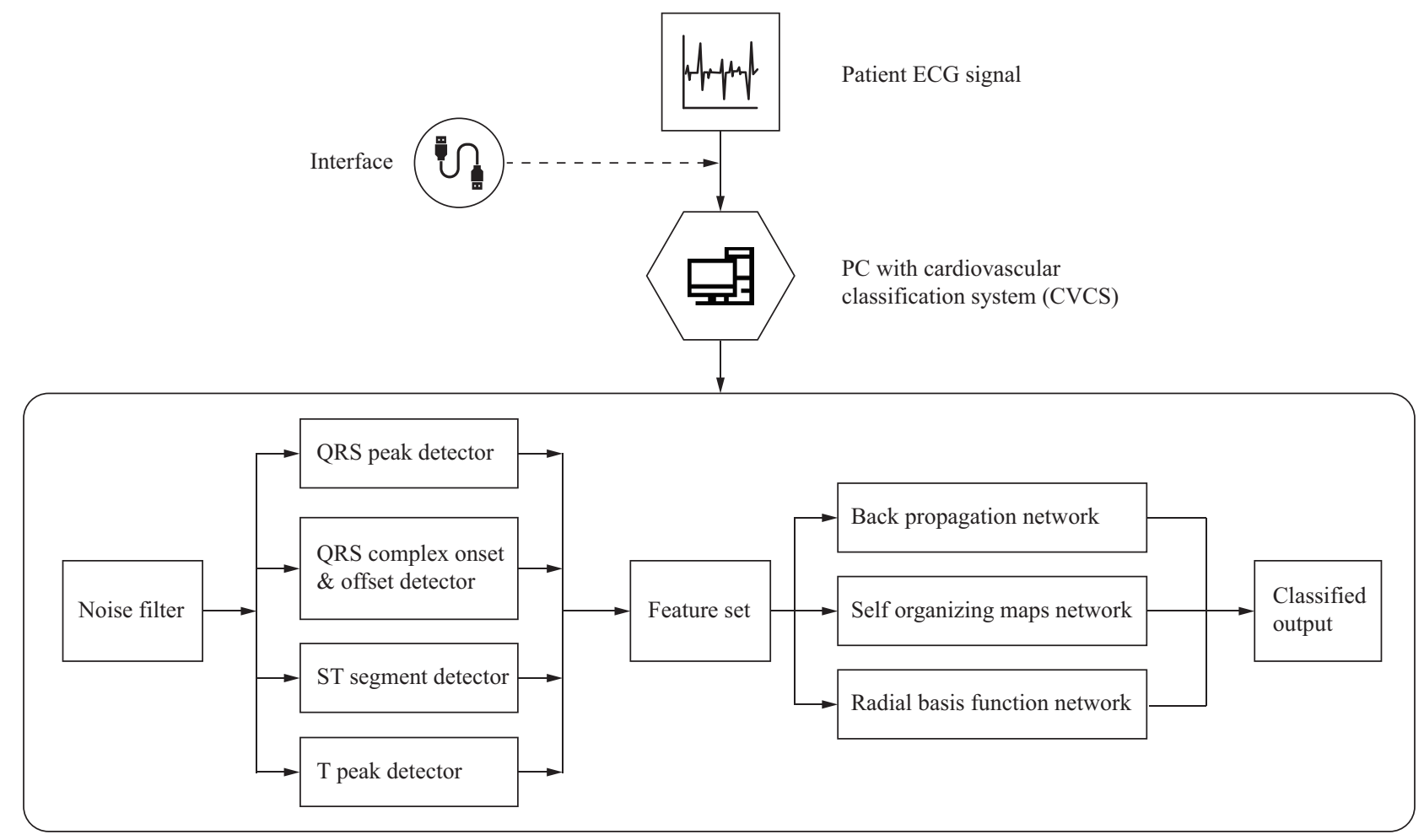

Fig. 4 Schematic diagram of the grading system for heart disease patients 
system to improve the prognosis of patients with myocardial infarction. The system formulated exercise programs according to patients' disease and lifestyle information and monitored patients' vital signs during exercise to estimate their exercise intensity and promote productive exercise habits. This in turn helped realize individualized guidance for patients' cardiac rehabilitation.

\subsection{Scenario-Based Applications of Emergency and First Aid}

Emergency treatment has always been faced with a relative lack of medical resources, including the need for rapid triage and treatment of emergency and critically ill patients, as well as the uneven level of diagnosis and treatment of medical staff. This directly points out that using AI and IoT technology to analyze and deal with a large number of emergency diagnosis and treatment data will provide important guidance for rapid and accurate diagnosis and treatment.

As an important part of the emergency medical system, pre-hospital first aid is of great significance for rescuing emergency and critically ill patients. This improves their prognosis and promotes recovery, indicating that it is very important to use advanced technology to carry out early warning and intervention in pre-hospital first aid. Chen et $a l^{[70]}$ predicted and visualized the needs of pre-hospital emergency medical care through the combination of machine learning algorithms and geographic information systems (GISs). In addition, we are also studying the use of IoT technology to collect patients' vital signs data, combined with electronic medical records and other information, to build an intelligent early-warning model to carry out real-time grading of the critical degree of patients in the ambulance.

In the prediction of early disease diagnosis, because of the heavy emergency work in the past, how to early and accurately identify the diagnosis and treatment intervention has been one of the most important tasks of the emergency department. Now, with the help of AI and other means, we can carry out early auxiliary diagnosis, improve the efficiency of diagnosis and treatment, and reduce the rate of missed diagnosis. Tsien et $a^{[71]}$ adopted machine learning and other techniques to screen acute myocardial infarction in patients with emergency chest pain. Green et al ${ }^{[72]}$ also constructed a prediction model of emergency acute coronary syndrome using a neural network.

The prediction of disease prognosis in emergency work is also important in clinical work. Bentley et al ${ }^{[73]}$ analyzed the head CT of patients with ischemic stroke using AI technology, and predicted the outcome of cerebral hemorrhage after thrombolysis.

In order to deal with the occurrence of public emergencies such as COVID-19 pandemic, it is necessary to establish a monitoring and early warning mechanism for public health emergencies. We also need to make use of the combination of IoT, AI and emergency rescue to further improve the early warning mechanism for major disasters, improve the medical treatment security system and build an intelligent decision-making system. Toltzis et al ${ }^{[74]}$ built a corresponding model through AI technology, which identified children who needed to be admitted to the intensive care unit for a short time among patients with mass casualties caused by disasters. France et a ${ }^{[75]}$ deduced the quantitative indicators and optimization methods of the hospital's ability to cope with the surge of patients after disasters through computer simulation technology and other tools, which contributed to the improvement of hospital disaster emergency management capacity.

\subsection{VTE Scenario Application}

Venous thromboembolism (VTE) is a global medical problem. The risk of VTE in a hospital is related to the patient's condition, operation, etc. Multicenter studies show that the VTE prevention rate among Chinese inpatients is only $6.0 \%$ in internal medicine and $11.8 \%$ in surgery ${ }^{[76]}$. By contrast, an international cross-sectional study showed that the VTE prevention rate was $39.5 \%$ in internal medicine and $58.5 \%$ in surgery ${ }^{[77]}$. There are significant differences in the level of prevention between China and other countries in the world. About $5 \%-10 \%$ of hospitalized deaths in China are caused by VTE. Therefore, early identification of high-risk patients with VTE and timely intervention can significantly reduce the incidence of hospital VTE.

Based on the Caprini and Padua evaluation scales in Peking University Cancer Hospital, Wang et al ${ }^{[78]}$ applied big data tools to extract the scoring index in real-time using intelligent control to automatically evaluate, push and detect early warning signs in terms of these scoring scale. This approach significantly improved the VTE evaluation rate of tumor patients. The VTE intelligent risk assessment technology based on big data technology has raised the in-hospital VTE evaluation rate from $11 \%-87 \%$ by hand to $100 \%$ by machine. This incorporated the full Caprini evaluation of surgical patients and the full Padua evaluation of internal medicine patients.

With the help of the information system, Meng et $a{ }^{[79]}$ constructed a VTE prevention and treatment system based on big data and AI. In the daily use of the system, it helped medical staff evaluate and manage patients through pre-reminders, automatic filling, and clinical decision-making. Xuanwu Hospital, Capital Medical University, through the construction of a VTE prevention and treatment system, has demonstrated that the quality control of VTE prevention and treatment can be detected before and counted during the event.

\subsection{Scenario-Based Application in Custodial Care}

A monitoring system is an important tool for a 
hospital to monitor patients. These systems use AI, IoT, big data and other technologies, including bedside monitoring equipment, acquisition and transmission equipment and monitoring software. The main function of these systems is to collect, calculate and present the patient's vital sign parameters in real-time, and to generate alarms by identifying abnormal values to improve the level of monitoring and nursing in many ways.

Vitalerter, an Israeli digital health brand, has designed a contactless continuous monitoring and early warning protection program based on advanced biosensors and deep learning to provide continuous and contactless vital sign monitoring and cloud-based early warning protection services for long-term care of patients. Exemplary features of these systems include the contactless detection of patients out of bed, the continuous measurement of heart and respiratory rate, and the accurate analysis of body movement. In case of adverse events, the system will automatically issue a voice alarm to remind nurses to intervene immediately and reduce the risk of falls, pressure sores and septicemia. Fig. 5 shows a schematic diagram for monitoring the vital signs of patients in a non-contact manner ${ }^{[80]}$.

In the practical application of a monitoring system, noise and pseudo-error interference, weak sensors and unreasonable threshold settings often lead to excessive false-positive and false-negativeal alarms, which lead to alarm fatigue for medical staff. The American Academy of Emergency Medical Research has listed alarm fatigue as one of the "top ten medical technical hazards' ${ }^{[81]}$. Alarm fatigue reduces the attention and response speed of nurses to the real critical alarm, who may eventually ignore or even turn off the alarm completely. These behaviors pose a significant threat to the life and safety of patients.

Clinical studies have confirmed that the parameter threshold of a monitor cannot be set reasonably based on the patient's condition and has become one of the common factors contributing to alarm fatigue. All the

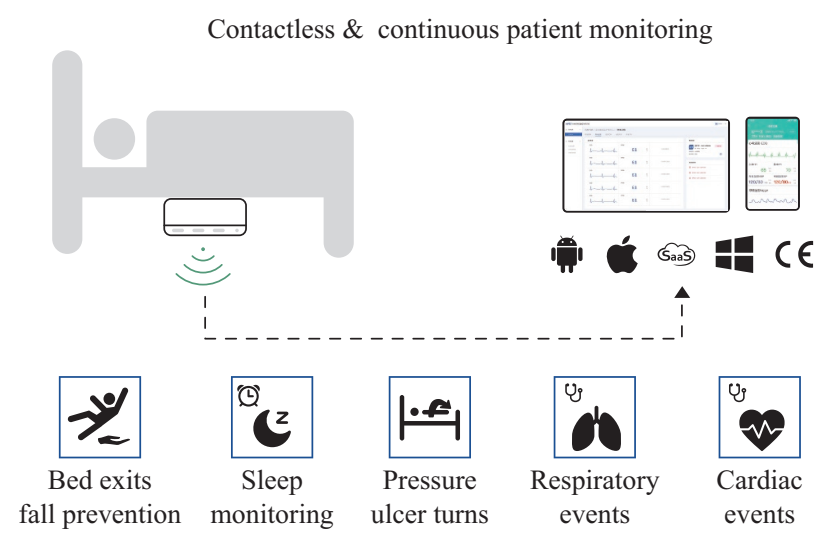

Fig. 5 Schematic diagram of a non-contact patient's vital signs monitoring program parameters of the monitoring system are equipped with default thresholds, but these default values can not meet the individual needs of the disease, and often result in a large number of worthless alarms ${ }^{[82]}$. Therefore, improving the rationality and ease-of-use of the threshold setting is of great significance to alleviate alarm fatigue. Neusoft Hanfeng collects the data of the monitor in real-time through an IoT acquisition terminal, independently calculates and analyzes the physical parameters of each patient, establishes an independent alarm threshold for each patient according to the patient's personal condition, and dynamically modifies the threshold interval through continuous monitoring to greatly reduce the occurrence of an invalid alarm.

According to an ICU alarm survey in Germany, $87 \%$ of health care workers believe that less than half of the alarms need to be dealt with, and $52 \%$ think that less than $25 \%$ of the alarms are of clinical significance ${ }^{[83]}$. Short-term transient threshold overlimit alarms are often caused by patients turning over, coughing or other reasons, and most of these alarms do not need to be treated. Therefore, in view of this clinical actual situation, Dongsoft Hanfeng developed the alarm delay function and classified the alarm situations. For the important alarm, it was triggered immediately. By contrast, for the secondary alarm, if the information that falls back to a normal level immediately after exceeding the threshold, or if the information that exceeds the threshold is not high, it will carry out delayed trigger or de-trigger processing.

\subsection{Clinical Application of Medical Imaging- Assisted Diagnosis}

Medical imaging data account for up to $90 \%$ of clinical medical data, and intuitive and clear data is the key factor affecting clinical diagnosis. AI is widely used in intelligent image recognition, intelligent assistant personalized diagnosis, human-computer interaction aided diagnosis, precision treatment assistant decisionmaking, etc.

Google's Gulshan team ${ }^{[84]}$ developed a deep learning algorithm for detecting diabetic retinopathy and diabetic macular edema. This same team and ophthalmology experts collected more than 120000 retinal fundus images, trained a CNN algorithm model using part of the image data, and then intelligently classified the test image data for verification and evaluation. According to the results, this model exhibited good performance in the detection of diabetic retinopathy. In 2016, the results were published in JAMA, with this model providing an important reference for the American Food and Drug Administration (FDA) to review the diagnostic equipment of "diabetic retinopathy AI" in the United States.

The Esteva team of Stanford University ${ }^{[85]}$ collected 
about 130000 images of skin lesions obtained by dermoscope scanning through the online dermatology information database, the International Skin Imaging Collaboration (ISIC) dermatoscope archives, the Edinburgh Library and Stanford Hospital. These images were marked, classified and verified by dermatologists to contain lesions from 2032 different diseases. Using these data, the Esteva team trained a CNN for skin cancer classification. After testing, the sensitivity and specificity of the model were comparable to the diagnostic level of dermatologists.

Zhang Kang's team ${ }^{[86]}$ at Macau University of Science and Technology has developed an AI-based COVID-19 diagnosis system, which is based on lung CT images and can accurately diagnose, quantify and predict COVID-19. The system consisted of two parts, a lung lesion segmentation module and the diagnosis analysis module. Zhang Kang's team selected more than $43 \mathrm{CT}$ images from the database of the China chest CT Imaging Research Union, and trained, crossvalidated and tested the system using these image data. Tests showed that the system exhibited superior sensitivity and specificity compared with the diagnosis of radiologists, and that the system reached the diagnostic level of middle and senior doctors.

\section{CHALLENGES AND TRENDS}

\subsection{Challenges}

With the integration of AI, IoT and the medical industry, intelligent medical IoT demonstrates various new features. IoT connection terminals have a large number of data modes that come in various forms and exhibit cross-domain fusion characteristics. Therefore, this field needs multi-disciplinary knowledge fusion, such as medicine, AI, big data, information engineering, etc. However, new features bring new challenges and smart medical IoT faces complex risks and challenges. 5.1.1 Information Security is Threatened The number of intelligent medical IoT terminals is huge and they are deployed in clusters. Attackers can easily launch distributed denial of service attacks through violent cracking and by sending malicious data packets. These attacks result in network congestion, paralysis, service interruption, and are a threat to the diagnosis and treatment processes as well as the life and safety of patients.

Intelligent medical IoT collects a large amount of group life and health data, and its infrastructure includes virtual machines, cloud platforms, databases, middleware, web applications, etc. Due to software design, process design and other factors, there are security risks such as authentication bypass, unauthorized access, data tampering, remote control, service interruption, and more. Abdallah Soualmi et $a l^{[87]}$ confirmed that there is a risk of affecting the privacy and security of user data through the use of services and applications over the internet, and that unauthorized parties may steal, use, modify or destroy sensitive data.

5.1.2 IoT Data Standards are Missing Due to the large number of intelligent medical IoT equipment manufacturers, the quality of IoT terminal products is difficult to be effectively guaranteed, which brings the challenge of ensuring the reliability and quality of data $^{[88]}$. However, the massive training set formed by IoT equipment-based group life and health data plays an important role in the accuracy of AI algorithms such as machine learning. Farhad Ahamed et al[89] mentioned that the data sets generated by IoT terminals may sometimes be biased and cannot fully cover all medical scenarios, which may greatly reduce the accuracy and effectiveness of intelligent diagnosis, and can eventually lead to the deviation of "personalized medical care".

At present, some progress has been made in IoT standardization, but it mainly focuses on architecture and security technology, including encryption lightweight, authentication, privacy control, etc. In the aspect of traditional medical informatization, although HL7 and other medical standardization organizations have issued reference information models such as Reference Information Model (RIM), V2.X, V3.0, Certified Data Analyst (CDA), etc., their applications mainly focus on traditional clinical information and are not compatible with IoT information and medical image data, multi-text data, waveform data, etc.

\subsubsection{Risks Brought by the Refinement of Specialties}

The refinement of specialties is a major feature of modern medicine. Although targeted research on a disease or a specific medical field can effectively improve the rate of diagnosis and treatment, once a specialist is too detailed, doctors lack the overall concept of diagnosis and treatment. This scenario can easily lead to misdiagnosis. At present, the research of medical AI is often based on a certain disease to carry out research and clinical practice in a certain specialty. Such practice precisely initializes the subdivision of modern medicine in the field of AI so that its accurate diagnosis ability is affected. Medical AI should be oriented to the whole medical scene, and account for the multi-disciplinary, multi-modal and full diagnosis and treatment information to achieve an accurate medical goal.

5.1.4 Impact of AI on Ethics The emergence of AI in the medical field has had an impact on traditional medical ethics to a certain extent. It is difficult for patients to accept the computer to give advice on disease prevention, diagnosis, treatment and rehabilitation instead of doctors. This results in a reduction of patients' trust in doctors, which may lead to a series of doctor-patient conflicts and even legal 
disputes. At the same time, medical decision-making is more complex, and the effect of AI applications are closely related to the quality and coverage of training data sets and the advanced nature of AI algorithms. It is difficult to consider factors that are difficult to quantify, such as patients' economic affordability, patients' psychological tolerance to diagnosis and treatment plans, and different social habits in different regions of the world. Therefore, the decision made by AI may not be the optimal solution for patients after combining various factors. By contrast, it is one-sided and the decision-making process of AI requires the participation of medical experts.

\subsubsection{Medical Compound Talents are Lacking}

Extracting knowledge from massive life and health data, and achieving AI through clinical verification requires in-depth clinical analysis, information modeling, data extraction, AI algorithm construction, software and hardware research and development, clinical experiments and other processes. This also requires researchers to have deep expertise in both medical and technical fields, which are also knowledge-intensive industries. Although medical big data and medical AI are gradually on the rise, it is a pity that there are very few personnel who understand the characteristics of these two industries. In recent years, the education system has been aware of the lack of compound talent, and has carried out the construction of interdisciplinary talent training systems that try to solve this problem from the establishment of educational disciplines.

\subsection{Trends}

With the continuing development of AI and IoT technologies, the future of AI and IoT will be more widely and deeply applied to the medical field.

\subsubsection{AIoT is Entering a Period of Rapid Development}

AIoT, namely "AI+IoT", is the inevitable outcome of the integration of AI technology and IoT, and is the advanced form of the intelligent development of IoT. AI and IoT are symbiotic technologies where IoT collects, transmits and processes real-world data through a variety of devices. AI complements IoT by mining and analyzing this data to increase the value of IoT. In recent years, the construction of smart medicine has provided the most direct application scenarios and data sources for AIoT, and the breakthrough of AI technology in the medical field has directly promoted the development of AIoT. With the outbreak of the COVID-19 pandemic, AIoT and big data technology have played a key role in epidemic tracking and antiepidemic technologies, promoting the rapid integration of a large number of AIoT scenarios. More applications are expected to continue to be popularized in the next decade. Fig. 6 shows the global market scale of AIoT in 2019 and $2022^{[90]}$.

5.2.2 AI is Becoming More Common on Edge Devices Currently, most IoT applications that use AI are implemented in the cloud because the technology of medical IoT is limited ${ }^{[91,92]}$. In the future, more and more medical devices, from the examination and medical images, operating rooms, ICU, emergency departments, wards, and even wearable community and family health devices, will be connected to IoT terminals to form massive medical data. Edge computing has the advantages of strong practicability, ease-of-use and deployability, so it is embedded in the IoT access terminal of medical equipment for localized data computing, storage and edge intelligent services. In the near future, the IoT terminal products of medical devices with edge computing capabilities will become more and more mature. In addition, these devices will continue to emerge in hospital diagnosis and treatment plans, post-hospital rehabilitation and daily health management, which will contribute to the development of remote monitoring, medical imaging, telemedicine and other services. Fig. 7 shows the application of the fusion between the cloud and edge devices in the medical field ${ }^{[93]}$.

5.2.3 Intelligent Monitoring, Early Warning and Intelligent Diagnosis Technology Applied in the Field of Infectious Diseases Some developed countries have currently begun to explore the application of

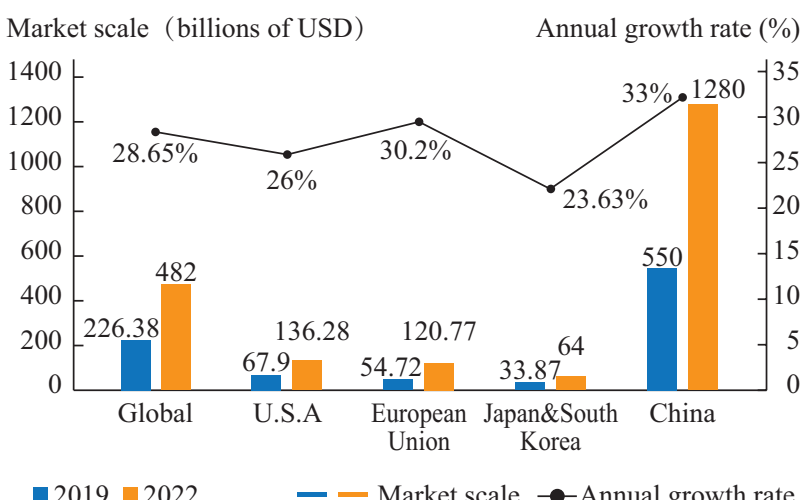

Fig. 6 Global market scale of AIoT in 2019 and 2022 AIoT: AI+IoT

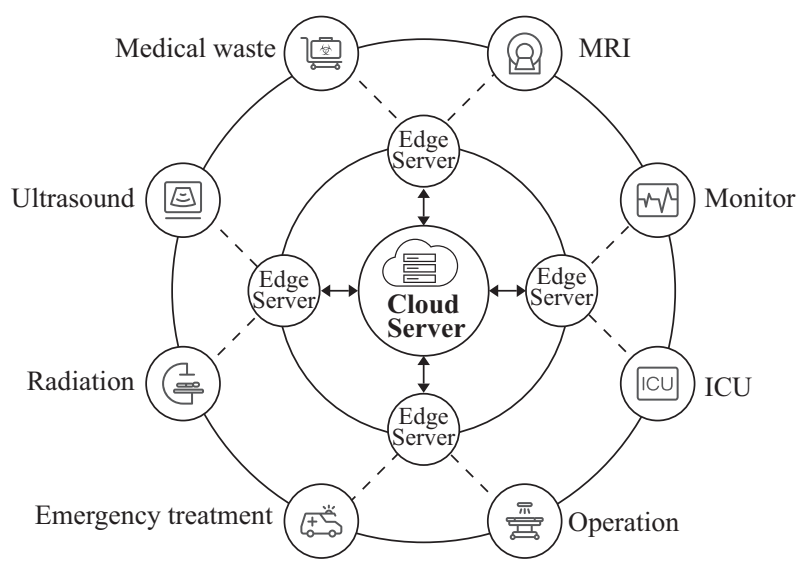

Fig. 7 The application of the fusion of the cloud and edge in the medical field 
pathogen and genome information integration in infectious disease surveillance and early warning ${ }^{[94}$, ${ }^{95]}$. The etiology, environment and sociology related to the spread of big infectious disease data are applied to carry out multivariate AI management analysis and early pandemic warning. In the field of diagnosis, the efficiency of clinical infectious disease diagnosis has been greatly improved through AI diagnosis technology and equipment ${ }^{[96]}$. In the future, the application of AI diagnosis technology and equipment for infectious diseases will make great breakthroughs in automatic sample processing technology, as well as rapid, sensitive, specific and high-throughput pathogen detection technology. These advances will promote host-based biomarker detection and detection technology.

\subsubsection{More Robot Products Will Enter the Medical}

Scene With the aging world population, the demand of medical robots is gradually increasing, which has become one of the main application scenarios of AI in the medical field. A variety of medical robots with different functions have been developed, such as surgical robots, nursing robots, service robots, guide robots, logistics robots, rehabilitation robots, dispensing robots, etc. With the application and integration of AI, 5G and other cutting-edge technologies, medical robots will achieve new and major upgrades. The large-scale use of medical robots will be a trend in the future medical field. Fig. 8 shows the global turnover of medical robots in 2018-2019 and potential development in 2020-2023 ${ }^{[97]}$.

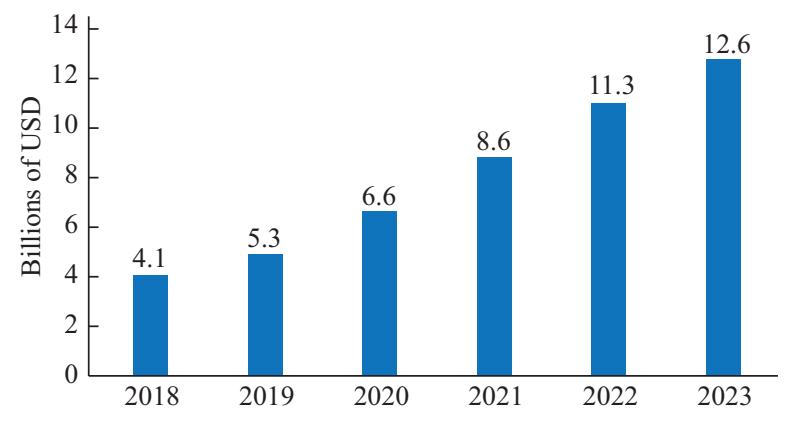

Fig. 8 The global turnover of medical robots in 2018-2019 and potential development in 2020-2023

\subsubsection{Future Medicine-Oriented Intelligent Decision} Support Systems Will be Implemented With the application and popularization of gene detection technology and intelligent wearable devices, real-world data will be more and more complete. The traditional clinical medicine model will change greatly, and future treatment planning will be tailored to the information of individual patients. The intelligent decision support system with comprehensive use of IoT, big data, AI and other technologies will provide important support for the development of medicine in the future. The application scope of the new generation of intelligent decision support systems will include in-hospital, outof-hospital, medical group, regional medical and other comprehensive applications. The application forms will gradually integrate with the medical information system and will be applied to current auxiliary diagnosis and treatment as well as other individual clinical applications such as medical and health. These applications will help to achieve comprehensive coverage of personal health management, medical services, medical quality management, operation and maintenance, clinical scientific research, pharmaceutical equipment research and development.

\section{CONCLUSIONS}

In this paper, all data was collected and transferred to the cloud or another space using IoT, while algorithms and methods such as AI activity identification, behavior identification, abnormal detection, and assisted decision-making were used to gain meaningful insights from the collected data to inform decision making.

The integration of AI and IoT allows IoT to gain advanced intelligence, and has begun to be used in clinical medicine to make health care more intelligent. Remote diagnosis and treatment collaborations can accelerate the equalization of diagnosis and treatment services. Combined with medical IoT, the real-time vital sign data of patients can be obtained, and an AI model algorithm can be used to identify the early risk of NICU and CCU patients, recommend treatment plans and predict the prognosis of treatment. In emergency care, AI and IoT can be used to provide rapid and accurate diagnosis and treatment, which can buy time for rescue and open the green life channel. Early identification of high-risk patients with VTE and timely intervention can reduce the incidence of VTE and improve the quality-of-life of patients. Real-time presentation of patients' vital sign data and intelligent alarms by identifying outliers can effectively improve the monitoring level and ensure the safety of patients. AI enables medical image recognition and diagnosis to be more accurate and efficient.

In the future, AI and IoT will play a role in all aspects of hospital management and service. With the help of IoT, electronic tags customized for medical equipment assets based on QR codes, RFID and other technologies improve the coincidence rate of accounts and materials, and realize real-time status monitoring of equipment through the deployment of IoT sensing terminals. Importantly, this will help to achieve comprehensive and objective management of medical equipment. The supervision and management of the whole process of medical waste have promoted the link between the collection, classification, packaging and storage of medical waste in medical and health 
institutions and the later treatment and management of medical waste. This enables the standardization, systematization, scientification and modernization of medical waste supervision of medical institutions. Technology will continue to create more new models for smart medicine, smart management and smart services, and contribute to the high-quality development of medical care.

\section{Conflict of Interest Statement}

None of the authors have any conflict of interest.

\section{REFERENCES}

1 GHWA/WHO. A Universal Truth: No Health Without a Workforce

2 WHO. State of the World's Nursing Report 2020

3 Kruk ME, Gage AD, Joseph NT, et al. Mortality due to low-quality health systems in the universal health coverage era: A systematic analysis of amenable deaths in 137 countries. Lancet, 2018,392(10160):2146-2147

4 World Population Prospects 2019: Highlights[B]. ONU. United Nations. 2019

5 Healthy Aging Team. The Top 10 Most Common Chronic Conditions in Older Adults.National council on ageing. Available from: https://dailycaring.com/ prevent-and-manage-the-10-most-common-chronicdiseases-in-older-adults/

6 Jaul E, Barron J. Age-Related Diseases and Clinical and Public Health Implications for the 85 Years Old and Over Population. Front Public Health, 2017,5:335-335

7 van den Bussche H, Koller D, Kolonko T, et al. Which chronic diseases and disease combinations are specific to multimorbidity in the elderly? Results of a claims data based cross-sectional study in Germany. BMC Public Health, 2011,11:101

8 Mofizul IM, Valderas JM, Laurann Y, et al. Multimorbidity and Comorbidity of Chronic Diseases among the Senior Australians: Prevalence and Patterns. Plos One, 2014,9(1):e83783

9 Zhao C, Liping W, Zhu Q, et al. Prevalence and correlates of chronic diseases in an elderly population: A community-based survey in Haikou. Plos One, 2018, 13(6):e0199006

10 Burroughs A. What Is a Tele-ICU and How Does It Work? Available from https://healthtechmagazine.net/ article/2020/09/what-tele-icu-and-how-does-it-work

11 Fuller T, Fox B, Lake D, et al. Improving real-time vital signs documentation. Nurs Manage. 2018,49(1):28-33

12 Martine L. Measuring patient and clinical effectiveness. Microsoft Industry Blogs - United Kingdom Available from: https://cloudblogs.microsoft.com/industry-blog/ en-gb/health/2020/07/03/measuring-patient-andclinical-effectiveness/

13 Prajapati B, Parikh S, Patel J. An Intelligent Real Time IoT Based System (IRTBS) for Monitoring ICU Patien. International Conference on Information and Communication Technology for Intelligent Systems. Springer, Cham, 2017

14 Hka F, Swk B, Ep C, et al. The role of fifth-generation mobile technology in prehospital emergency care: An opportunity to support paramedics. Health Policy
Technol, 2020, 9(1):109-114

15 Tang $X$. The role of artificial intelligence in medical imaging research. BJR Open, 2019,2(1):20190031

16 Chamberlin J, Kocher MR, Waltz J, et al. Automated detection of lung nodules and coronary artery calcium using artificial intelligence on low-dose CT scans for lung cancer screening: accuracy and prognostic value. BMC Med, 2021,19(1):55

17 Wang XN, Dai L, Li ST, et al. Automatic Grading System for Diabetic Retinopathy Diagnosis Using Deep Learning Artificial Intelligence Software. Curr Eye Res, 2020,45:1550-1555

18 Dey D, Slomka PJ, Leeson P, et al. Artificial Intelligence in Cardiovascular Imaging. J Am Coll Cardiol, 2019, 73(11):1317-1335

19 Alkhatib H, Faraboschi P, Frachtenberg E, et al. IEEE CS 2022 Report. IEEE Computer Society, 2014:25-27

20 Kosmatos EA, Tselikas ND, Boucouvalas AC. Integrating RFIDs and Smart Objects into a UnifiedInternet of Things Architecture. Adv Internet Things, 2011,1(1):5-12

21 Madakam S, Ramaswamy R, Tripathi S. Internet of Things (IoT): A Literature Review. J Comp Commun, 2015,3(3):164-173

22 Gubbi J, Buyya R, Marusic S, et al. Internet of Things (IoT): A Vision, Architectural Elements, and Future Directions. Future Gener Comp Syst, 2013,29(7):16451660

23 Haider F. Cellular architecture and key technologies for $5 \mathrm{G}$ wireless communication networks. J Chongqing Univ Posts Telecommun, 2014,52(2):122-130

24 Joyia GJ, Liaqat RM, Farooq A, et al. Internet of medical things (IOMT): Applications, benefits and future challenges in healthcare domain. J Commun, 2017,12(4):240-247

25 Hingmire M, Bagjilewale M, Dakhole M. What is Cloud Computing. Springer Verlag Ny, 2017,17(1): 3-20

26 Sultan N. Making use of cloud computing for healthcare provision: Opportunities and challenges. Int J Inform Manage, 2014,34(2):177-184

27 Wang L, von Laszewski G, Younge A, et al. Cloud Computing: a Perspective Study. New Generat Comput, 2010,28(2):137-146

28 Ahuja SP, Sindhu M, Jesus Z. A Survey of the State of Cloud Computing in Healthcare. Network Commun Technol, 2012,1(2)12-19

29 Marjani M, Nasaruddin F, Gani A, et al. Big IoT Data Analytics: Architecture, Opportunities, and Open Research Challenges. IEEE Access, 2017,5(99):52475261

30 Kufrin R. Decision trees on parallel processors. Machine Intelligence Pattern Recognition, 1997,20:279-306

31 Gondy LA, Thomas C, Bayes N. Programs for machine learning. Advances in Neural Inform Proc Syst, 1993,79(2):937-944

32 Judith E, James M. Artificial neural networks. Cancer, 2001,91(S8):1615-1635

33 Krallinger M, Leitner F, Vazquez M, et al. Text Mining. Compr Biomed Phys, 2014,6(10 Supplement):51-66

34 Quan XX, Yang J F, Luo Z. Models in digital business and economic forecasting based on big data IoT data visualization technology. Pers Ubiquit Comput, 
2021(https://doi.org/10.1007/s00779-021-01603-7)

35 Hua X, Aldrich MC, Chen Q, et al. Validating drug repurposing signals using electronic health records: a case study of metformin associated with reduced cancer mortality. J Am Med Inform Assoc, 2015(1):179-191

36 Dash S, Shakyawar SK, Sharma M, et al. Big data in healthcare: management, analysis and future prospects. J Big Data, 2019,6(1):54

37 Bhardwaj R, Nambiar AR, Dutta D. A Study of Machine Learning in Healthcare. 2017 IEEE 41st Annual Computer Software and Applications Conference (COMPSAC). July 4-7, 2017, Turin, Italy

38 Abramson N, Braverman DJ, Sebestyen GS. Pattern Recognition and Machine Learning. Public Am Statist Assoc, 2006,103(4):886-887

39 Avci A, Bosch S, Marin-Perianu M, et al. Activity Recognition Using Inertial Sensing for Healthcare, Wellbeing and Sports Applications: A Survey. ARCS'10 - 23th International Conference on Architecture of Computing Systens 2010, Workshop Proceedings, February 22-23, 2010, Hannover, Germany. VDE, 2010

40 Ijjina EP, Mohan CK. Hybrid deep neural network model for human action recognition. Appl Soft Comput, 2016:936-952

41 Goodfellow I, Bengio Y, Courville A. Deep learning. Cambridge: MIT Press, 2016:367-415.

42 Liu X, Yang XD. Multi-stream with deep convolutional neural networks for human action recognition in videos. Neural Information Processing. Cham: Springer International Publishing, 2018:251-262.

43 Wang LL, Ge LZ, Li RF, et al. Three-stream CNNs for action recognition. Pattern Recog Lett, 2017,92:33-40

44 Tran D, Bourdev L, Fergus R, et al. Learning Spatiotemporal Features with 3D Convolutional Networks. 2015 IEEE International Conference on Computer Vision (ICCV), December 7-13, 2015, Santiago, Chile, IEEE, 2015:4489-4497

45 Qiu Z, Yao T, Mei T. Learning Spatio-Temporal Representation with Pseudo-3D Residual Networks. 2017 IEEE International Conference on Computer Vision (ICCV), October 22-29, 2017, Venice, IEEE, 2017:5533-5541

46 Zhou Y, Sun X, Zha ZJ, et al. MiCT: Mixed 3D/2D Convolutional Tube for Human Action Recognition. 2018 IEEE/CVF Conference on Computer Vision and Pattern Recognition (CVPR), June 18-23 2018, Salt Lake City, UT, IEEE, 2018:449-458

$47 \mathrm{Ng} \mathrm{YH}$, Hausknecht M, Vijayanarasimhan S, et al. Beyond short snippets: Deep networks for video classification. 2015 IEEE Conference on Computer Vision and Pattern Recognition (CVPR), June7-12, 2015, Boston, MA, USA, IEEE, 2015:4694-4702

48 Du W, Wang Y, Yu Q. RPAN: An End-to-End Recurrent Pose-Attention Network for Action Recognition in Videos. 2017 IEEE International Conference on Computer Vision (ICCV), October 22-29, 2017, Venice, IEEE, 2017:3725-3734

49 Ren ZH, Xu HY, Feng SL, et al. Sequence labeling Chinese word segmentation method based on LSTM networks. Comput Appl Res, 2017,34(5):1321-1324

50 Wsy A, Syh B. A process-mining framework for the detection of healthcare fraud and abuse. Exp Syst Appl,
2006,31(1):56-68

51 Alabdulkarim A, Al-Rodhaan M, Al-Dhelaan TA. A Privacy-Preserving Algorithm for Clinical DecisionSupport Systems Using Random Forest. Comput Mater Contin, 2019,58(3):585-601

52 Tama BA, Lim S. A Comparative Performance Evaluation of Classification Algorithms for Clinical Decision Support Systems. Mathematics, 2020(8):1814

53 Patil K, Mohammad S. Big data privacy: A technological perspective and review. SSRN Electr J, 2017,4(11):159162

54 Abouelmehdi K, Beni-Hessane A, Khaloufi H. Big healthcare data: preserving security and privacy. J Big Data, 2018,5(1):1

55 Zhang DW, Li X, Jiang LX. New medical hotspot: remote collaborative diagnosis and treatment. Sci Technol Rev, 2017,35(10):26-31

56 Kulkarni A, Sathe S, Healthcare applications of the Internet of Things: A Review. Int J Comput Sci Inform Technol, 2014,5(5):6229-6232

57 Lu D, Tao L, The application of IOT in medical system. 2011 IEEE International Symposium on IT in Medicine and Education, December 9-11, 2011, Guangzhou, China, 272-275

58 Zhou WH, Xiao TT. Digital future of neonatal critical care medicine. Chin J Pediat (Chinese), 2021,59(4):261263

59 Barker DJ. Human growth and chronic disease: a memorial to Jim Tanner. Ann Hum Biol, 2012,39(5):335 341

60 Yang L, Liu X, Li Z, et al. Genetic aetiology of early infant deaths in a neonatal intensive care unit. J Med Genet, 2020,57:169-177

61 Yang L, Kong Y, Dong X, et al. Clinical and genetic spectrum of a large cohort of children with epilepsy in China. Genet Med, 2019,21(3):564-571

62 Pavel AM, Rennie JM, de Vries LS, et al. A machine learning algorithm for neonatal seizure recognition: a multicentre, randomised, controlled trial. Lancet Child Adolesc Health, 2020,4(10):740-749

63 Olga BL, Gao XM, Ehsan Y, et al. E-Healthcare: Remote Monitoring, Privacy, and Security. Microwave Symposium IEEE, December 12-14, 2014, Marrakech, Morocco

64 Masino AJ, Harris MC, Forsyth D, et al. Machine learning models for early sepsis recognition in the neonatal intensive care unit using readily available electronic health record data. PLoS One, 2019,14(2):e0212665

65 Sanchez Pinto LN, Stroup EK, Pendergrast T, et al. Derivation and validation of novel phenotypes of multiple organ dysfunction syndrome in critically ill children. JAMA NetwOpen, 2020,3(8):e209271

66 Kannathal N, Acharya UR, Lim CM, et al. Classification of cardiac patient states using artificial neural network. Exp Clin Cardiol, 2003,8(4):206-211

67 Sengupata PP, Huang YM, Bansal M, et al. Cognitive machine-learning algorithm for cardiac imaging: a pilot study for differentiating constrictive pericarditis from restrictive cardiomyopathy. Cire Cardiovasc Imaging, 2016,9(6):e004330

68 Schoenrath F, Markendorf S, Brauchlin AE, et al. Robotassisted training early after cardiac surgery. J Card Surg, 
2015,30(7):574-58

69 Ottavinano M, Vera-Munoz C, Arredondo MT, et al. Innovative self management system for guided cardiac rehabilitation. Conference proceedings: Annual International Conference of the IEEE Engineering in Medicine and Biology Society. IEEE Engineering in Medicine and Biology Society, August 30-September 3, 2011, Boston, USA, 2011:1559-1562

70 Chen AY, Lu TY, Ma MH, et al. Demand Forecast Using Data Analytics for the Preallocation of Ambulances. IEEE J Biomed Health Inform, 2016,20(4):1178-1187

71 Tsien CL, Fraser HS, Long WJ, et al. Using classification tree and logistic regression methods to diagnose myocardial infarction. Stud Health Technol Inform, 1998,52(1):493-497

72 Green M, Bjrk J, Forberg J, et al. Comparison between neural networks and multiple logistic regression to predict acute coronary syndrome in the emergency room. Artif Intell Med, 2006,38(3):305-318

73 Bentley P, Ganesalingam J, Carlton Jones AL, et al. Prediction of stroke thrombolysis outcome using CT brain machine learning. Neuroimage Clin, 2014,4: 635640

74 Toltzis P, Soto-Campos G, Shelton C, et al. Evidence Based Pediatric Outcome Predictors to Guide the Allocation of Critical Care Resources in a Mass Casualty Event. Pediatr Crit Care Med, 2015,16(7):e207-e216

75 Franc JM, Ingrassia PL, Verde M, et al. A simple graphical method for quantification of disaster management surge capacity using computer simulation and process-control tools. Prehosp Disaster Med, 2015,30(1):9-15

76 Zhai Z, Kan Q, Li W, et al. VTE risk profiles and prophylaxis in medical and surgical inpatients: The identification of Chinese hospitalized patients' risk profile for venous thromboembolism(DissolVE-2)-a cross-sectional study. Chest, 2019,155(1):114

77 Cohen AT, Tapson VF, Bergmann JF, et al. Venous thromboembolism risk and prophylaxis in the acute hospital care setting (ENDORSE study): a multinational cross-sectional study. Lancet, 2008,371(9610):387-394

78 Wang LJ, Pang J, Wang D, et al. FX. Design and construction of intelligent early warning system for venous thrombosis risk under big data technology. Chin Digit Med (Chinese), 2020,15(9):27-29

79 Meng Y, Li XY, Su JF, et al. Design and implementation of prevention and treatment system for venous thromboembolism (VTE). Chin Digit Med (Chinese), 2020,15(12):21-23

80 Integrated Care Platform[DB/OL]. [2021-09-22] https:// www.vitalerter.com/

81 ECRI Institute. Top 10 health technology hazards for 2020[EB/OL]. (2019-12-20)[2020-01-01] http://www. ecri.org

82 AACN. Practice alert: alarm management [EB/OL]. (2017-11-22). [2020-01-01] http://ccn.aacnjournals.org

83 Siebig S, Sieben W, Kollmann F, et al. Users'opinions on intensive care unit alarms-a survey of German intensive care units. Anaesth Intensive Care, 2009, 37(1): 112-116

84 Gulshan V, Peng L, Coram M, et al. Development and validation of a deep learning algorithm for detection of diabetic retinopathy in retinal fundus photographs. J Am Med Assoc, 2016,316(22):2402-2410

85 Esteva A, Kuprel B, Novoa RA, et al. Dermatologistlevel classification of skin cancer with deep neural networks. Nature, 2017,542:115-118

86 Zhang K, Liu XH, Shen J, et al. Clinically applicable AI system for accurate diagnosis, quantitative measurements, and prognosis of COVID-19 pneumonia using computed tomography. Cell, 2020,181(6):14231433

87 Soualmi A, Alti A, Laouamer L. Medical Data Protection Using BlindWatermarking Technique. Enabl AI Appl Data Sci, 2020:557

88 Tuli S, Tuli S, Wander G, et al. Next Generation Technologies for Smart Healthcare: Challenges, Vision, Model, Trends and Future Directions. Intern Technol Let, 2020,3:e145

89 Ahamed F, Farid F. Applying Internet of Things and Machine-Learning for Personalized Healthcare: Issues and Challenges. 2018 International Conference on Machine Learning and Data Engineering (iCMLDE),IEEE Computer Society, December 03-07, 2018, Sydney, Australia

90 Tuya Inc., Gartner Group. 2021 Global AIoT Developers Ecosystem White Paper. Tech Show Developers Conference, December 29, 2020, Hangzhou, China

91 Bangui H, Rakrak S, Raghay S, et al. Moving to the Edge-Cloud-of-Things: Recent Advances and Future Research Directions. Electronics, 2018,7(11):309

92 Alaybeyi S, Lheureux B. Survey Analysis: Artificial Intelligence Establishes a Foothold in IoT Projects. Gartner, Research, September 20, 2019. https://www. gartner.com/en/documents/3968034/survey-analysisartificial-intelligence-establishes-a-fo

93 Zhou Z, Shuai YU, Chen X. Edge intelligence:a new nexus of edge computing and artificial intelligence. Big Data Res, 2019,5(2):53-63

94 Ferdinand AS, Kelaher M, Lane CR, et al. An implementation science approach to evaluating pathogen whole genome sequencing in public health. Genome Med, 2021,13(1):121

95 European Centre for Disease Prevention and Control. Monitoring the use of whole-genome sequencing in infectious disease surveillance in Europe. Stockholm: ECDC; 2018

96 Qiu T, Yang Y, Qiu J, et al. CE-BLAST makes it possible to compute antigenic similarity for newly emerging pathogens. Nat Commun, 2018,9(1):1772

97 World Robotics 2020 Report [DB/OL]. [2020-09-24] https://ifr.org/news/record-2.7-million-robots-work-infactories-around-the-globe

(Received Oct. 12, 2021; accepted Nov. 26, 2021) 